\title{
Early surgery can improve the outcomes of patients with severe tricuspid regurgitation undergoing tricuspid replacement
}

\author{
Kun Hua $^{1 \#}$, Rui Zhao ${ }^{2 \#}$, Zhan Peng ${ }^{1}$, Yunxiao Yang ${ }^{1}$, Osmanaj Florian ${ }^{1}$, Bin Mao ${ }^{1}$, Xiubin Yang ${ }^{1,2}$ \\ ${ }^{1}$ Department of Cardiovascular Surgery, Beijing Anzhen Hospital, Capital Medical University, Beijing Institute of Heart, Lung and Vessel Disease, \\ Beijing, China; ${ }^{2}$ Department of Cardiovascular Surgery, Fuwai Hospital, National Centre for Cardiovascular Diseases, State Key Laboratory of \\ Cardiovascular Disease, Peking Union Medical College and Chinese Academy of Medical Sciences, Beijing, China \\ Contributions: (I) Conception and design: R Zhao; (II) Administrative support: B Mao, X Yang; (III) Provision of study materials or patients: K Hua, \\ B Mao; (IV) Collection and assembly of data: Z Peng, Y Yang, O Florian; (V) Data analysis and interpretation: R Zhao; (VI) Manuscript writing: All \\ authors; (VII) Final approval of manuscript: All authors. \\ "These authors contributed equally to this work. \\ Correspondence to: Bin Mao, MD; Xiubin Yang, MD. Department of Cardiovascular Surgery, Beijing Anzhen Hospital, Capital Medical University, \\ Beijing Institute of Heart, Lung and Vessel Disease, Beijing, China. Email: maobinsurgeon@163.com; xiubinyang@yahoo.com.
}

Background: Tricuspid regurgitation (TR) usually remains asymptomatic for a long time, such that it is most often diagnosed at an advanced stage of right heart failure. The purpose of this study was to identify clinical characteristics and overall outcomes in patients with severe TR who received tricuspid valve replacement (TVR) at different clinical stages.

Methods: Between 1993 and 2018, 256 severe TR patients who received TVR alone or in combination with other procedures were assessed at Beijing Anzhen Hospital. Ninety-three patients underwent New York Heart Association (NYHA) class I/II operations (early surgery group), and the others underwent NYHA class III/IV operations. The primary outcome was in-hospital and long-term mortality. Clinical outcomes were evaluated by the Kaplan-Meier method and Cox regression models. Follow-up was conducted annually. Propensity score matching and overlap propensity score weighting were performed as sensitivity analyses.

Results: Postoperative complications, including low cardiac output ( $11.8 \%$ vs. $26.4 \%, \mathrm{P}<0.001)$, renal failure $(2.2 \%$ vs. $16.6 \%, \mathrm{P}<0.001)$, and bleeding $(3.2 \%$ vs. $11.7 \%, \mathrm{P}=0.037)$, were significantly lower in the NYHA class I/II group than in the NYHA III/IV group. Patients in the NYHA class III/IV group had a significantly higher incidence of in-hospital mortality $(18.4 \%$ vs. $5.4 \%, \mathrm{P}<0.001)$ and long-term mortality (33.7\% vs. $11.8 \%, \mathrm{P}=0.006$ ) after follow-up (median follow-up duration $=63$ months). The results indicated a consistently higher occurrence rate in the propensity score-matched cohort and overlap propensity score weighted analysis.

Conclusions: Consistent with the recent clinical trend to provide earlier and more aggressive TR intervention, our results indicate that surgery for severe TR patients should be considered before advanced heart failure develops, when patients are asymptomatic or mildly symptomatic (NYHA class I/II).

Keywords: Tricuspid regurgitation; New York Heart Association classification (NYHA classification); tricuspid surgery

Submitted May 12, 2021. Accepted for publication Aug 23, 2021.

doi: $10.21037 / \mathrm{cdt}-21-311$

View this article at: https://dx.doi.org/10.21037/cdt-21-311 


\section{Introduction}

Tricuspid regurgitation (TR) often occurs alongside other valvular diseases and has a significant impact on the clinical conditions of patients. TR usually remains asymptomatic for a long time and is often diagnosed at an advanced stage of right heart failure (RHF) (1). Severe TR has also been recognized as an independent risk factor for longterm mortality (2). The latest guidelines support surgical treatment for asymptomatic or mild patients with severe TR $(3,4)$. However, this strategy continues to be debated, as some experts insist that surgery does not improve long-term survival outcomes (5), while others insist that good outcomes are achievable for tricuspid valve replacement (TVR) in properly selected patients and that surgery should occur much more early in many patients (6). Even mild symptoms that occur in patients with severe degenerative mitral regurgitation before surgical referral are associated with deleterious changes in cardiac structure and function (7). Therefore, the objective of the present study was to determine whether delaying replacement until symptoms progress causes adverse cardiac changes and clinical outcomes in patients with severe TR.

We present the following article in accordance with the STROBE reporting checklist (available at https://dx.doi. org/10.21037/cdt-21-311).

\section{Methods}

\section{Study population}

This is a retrospective observational study. Eligible patients were those who had severe TR and underwent TVR alone or combined with other procedures between 1993 and 2018 at Beijing Anzhen Hospital. Whether the valve was replaced or not and the choice of prosthesis were decided by the surgeon. During the study period, the preferential strategy was to repair tricuspid valves in our center. The indications for TVR were as follows: reoperation for recurrent TR after previous TV repair; surgeon preference to avoid future reoperation related to recurrent functional TR; and organic tricuspid leaflet pathology precluding valve repair. Preoperative, intraoperative, and postoperative data were obtained by reviewing medical records, and medical records were used to verify operations, echocardiogram reports, and hospitalizations. The study was conducted in accordance with the Declaration of Helsinki (as revised in 2013) and was approved by the Institutional Review Board of Beijing Anzhen Hospital (NO.: 2020101X). Individual consent for this retrospective analysis was waived.

\section{Clinical outcomes and follow-up}

Definitions of the Society of Thoracic Surgeons were used to determine complications. Acute kidney injury (AKI) was defined by documented diagnoses on discharge summaries and consultation notes or changes in serum creatinine as per the Kidney Disease Improving Global Outcomes (KDIGO) criteria. Postoperative low cardiac output syndrome was defined as the need for mechanical support-such as an intra-aortic balloon pump (IABP) and extracorporeal membrane oxygenation (ECMO), or continuous inotropic infusion (epinephrine $>0.1 \mu \mathrm{g} / \mathrm{kg} / \mathrm{min}$, norepinephrine $>0.1 \mu \mathrm{g} / \mathrm{kg} / \mathrm{min}$ ) — to maintain systolic blood pressure to $>90 \mathrm{mmHg}$ after correction for all reversible causes, including electrolyte imbalance and volume status. Operative mortality was defined as death within 30 days of operation or during the same hospitalization period. Patient condition was assessed via telephone calls and scheduled clinic visits. The median follow-up duration was 63 months, and the follow-up extended to 21 years. The completeness of follow-up was $90 \%$ for overall survival.

\section{Statistical analysis}

Normality was assessed using the Shapiro-Wilk statistical test. Continuous variables with normal distribution were expressed as means with standard deviations, and these variables were compared using the $t$-test. The chi-square test or Fisher's exact test was used to assess categorical variables. The Mann-Whitney test was used to compare nonnormally distributed continuous data, which were expressed as medians with interquartile ranges (IQRs).

Given that patients with severe symptoms are more susceptible to underlying comorbidities, propensity score (PS) matching and overlap PS weighting were performed to address potential confounding factors. A PS between New York Heart Association (NYHA) classes I/II and III/ IV was estimated using a multivariable logistic regression model incorporating patient age, sex, body mass index (BMI), hypertension, diabetes mellitus, atrial fibrillation (AF) history, lung disease, stroke history, hepatitis C, functional TR, Ebstein's Anomaly, cardiac surgery history, ejection fraction (EF), and isolated TVR. PS matching was performed using the nearest neighbor matching algorithm with a caliper size of 0.2 logit PS standard deviation units and a 1:1 ratio, after which the overlap PS weighting method was performed, with 


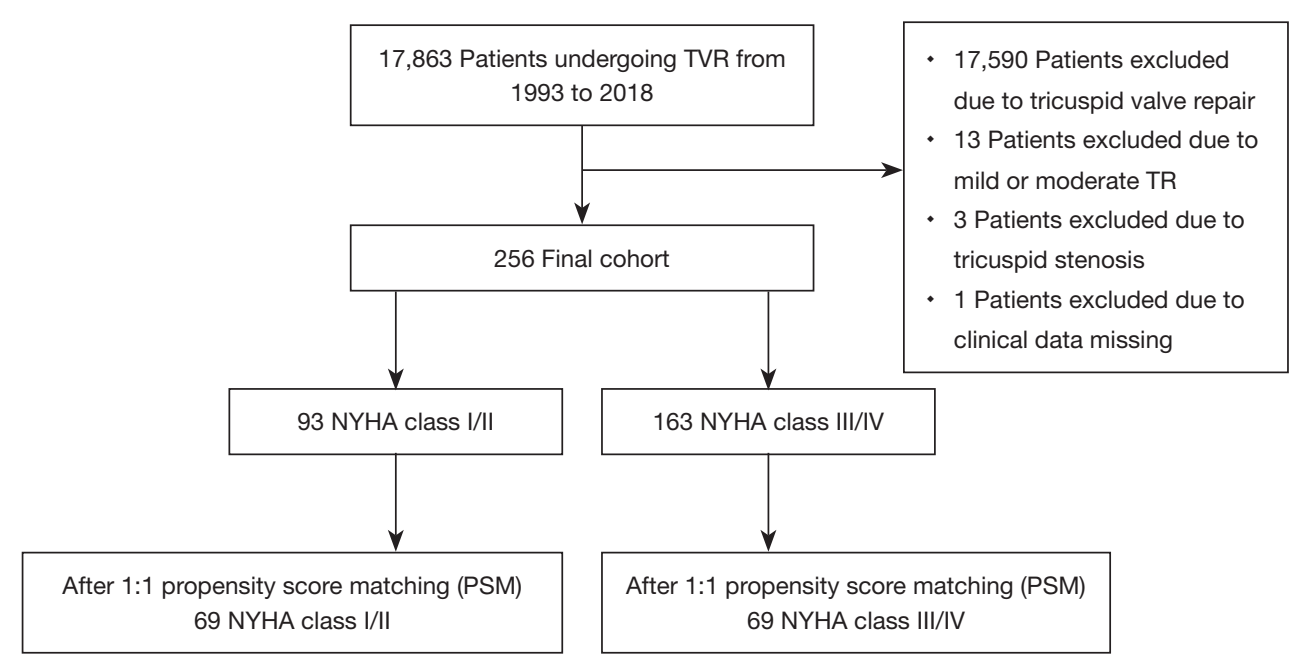

After overlap propensity score-weighted (OW) 46.59 NYHA class I/II

After overlap propensity score-weighted 46.59 NYHA class III/IV

Figure 1 Study design: summary of inclusion and exclusion criteria of study population. NYHA, New York Heart Association; TR, tricuspid regurgitation; TVR, tricuspid valve replacement.

the weight of each patient corresponding to the probability of that patient being assigned to the opposite group in this method (8). Overlap PS-weighted logistic regression models were used to analyze the association between symptoms severity and the probability of long-term survival, as well as other clinical outcomes. After PS matching and overlap PS weighing, the covariate balance was assessed using standardized mean differences, with values less than 0.2 reflecting adequate balance. We used the Kaplan-Meier method and the log-rank test to compare long-term followup survival rates between the NYHA I/II group and the NYHA III/IV group. Overall survival among NYHA classes was compared using unweighted and weighted log-rank tests. The adjustment covariates were those used to create the PS models, which were modeled using unadjusted and covariateadjusted Cox regression models.

All tests were two-tailed, and a $\mathrm{P}$ value $<0.05$ was considered significant. Analyses were performed using R, version 4.0.4 (https://www.r-project.org).

\section{Results}

\section{Perioperative characteristics in the original groups}

A total of 17,863 patients were referred for tricuspid valve surgery in Beijing Anzhen Hospital from 1993 to 2018. Of these, 256 patients were included in this study. Thirteen patients were excluded due to mild or moderate TR, and three patients were excluded due to tricuspid stenosis. One patient was ineligible because most clinical data were missing. Ninety-three patients who presented with NYHA class I/II were identified (Figure 1). Baseline characteristics by NYHA class are shown in Table 1. On average, NYHA class I/II patients were younger than those designated classes III/IV [41.00 (29.00, 51.00) vs. 49.00 (38.50, 57.50), $\mathrm{P}<0.001$ ], fewer NYHA class I/II patients were female, and fewer NYHA class $1 /$ II patients exhibited comorbidities such as histories of AF and previous valve surgery. Significantly fewer NYHA class I/II patients exhibited functional or rheumatic TR compared to those in class III/ IV ( $16.1 \%$ vs. $34.4 \%, \mathrm{P}=0.003 ; 30.1 \%$ vs. $65.6 \%, \mathrm{P}<0.001$, respectively). Additionally, patients in the NYHA class I/II group exhibited fewer signs of RHF, such as liver and spleen enlargements, ascites, and lower extremity edema.

\section{Intraoperative information and clinical outcomes}

Surgical information and clinical outcomes are presented in Table 2. There was no significant difference in intraoperative information between the two groups, including the 
proportion of patients with isolated TVR, cardiopulmonary bypass (CPB) time, whether to carry out aortic cross clamping (ACC), ACC time, and the type of prosthetic valve. There was a significantly lower prevalence of postoperative complications, including low cardiac output (11.8\% vs. 26.4\%, $\mathrm{P}<0.001)$, renal failure $(2.2 \%$ vs. $16.6 \%$, $\mathrm{P}<0.001)$, and bleeding ( $3.2 \%$ vs. $11.7 \%, \mathrm{P}=0.037)$, in the NYHA class I/II group than in the NYHA III/IV group. The same trend also occurred with regard to mechanical ventilation time $[16.00(13.00,25.00)$ vs. 24.00 (18.00, 47.00) hours, $\mathrm{P}<0.001]$ and intensive care unit (ICU) time [23.00 (16.00, 44.00) vs. $43.00(21.00,86.00), \mathrm{P}<0.001]$. Reoperation, reintubation, tracheostomy, liver failure, perioperative stroke, gastrointestinal bleeding, lower limb ischemia, and the use of IABP, ECMO, and CRRT were similar between the two groups. Patients in the NYHA class III/IV group were significantly more likely to experience inhospital mortality (18.4\% vs. $5.4 \%, \mathrm{P}<0.001)$. Additionally, in subsequent follow-up (median duration 63 months for the NYHA class III/IV group, IQR, 0-115 months vs. 65 months for NYHA class I/II group, IQR, 24-109 months), the NYHA class III/IV group showed significantly increased mortality (33.7\% vs. $11.8 \%, \mathrm{P}=0.006)$. The Kaplan-Meier curve for long-term prognosis is shown in Figure 2. We additionally explored whether early surgery based on NYHA classification was associated with overall mortality in the univariable Cox regression model, and the result was positive [NYHA I/II $v$ s. III/IV; hazard ratio (HR) $0.35,95 \%$ confidence interval (CI), 0.19 to $0.68, \mathrm{P}=0.002]$ (Table 3).

\section{PS-matched and overlap PS-weighted groups: characteristics and late outcomes}

Although this is a retrospective study based on the real world, we matched 69 pairs of patients to control for baseline differences and corroborate results. Cox regression models adjusting for variables used in constructing the PS models suggest there may be significant overall survival differences between NYHA patient classes, although our comparisons did not demonstrate statistical significance (NYHA I/II vs. III/IV; HR 0.57, 95\% CI, 0.27 to 1.24 , $\mathrm{P}=0.156)$. A decreased sample size might weaken statistical power after PS matching, and not all covariates were well balanced (Figure 3). Given that there is a better PS method that exactly balances the mean of every measured covariate, we further assessed our data using the overlap PS weighting method and the same covariates used in PS matching (8). After overlap PS weighting, the standardized differences between almost all the covariates were $<10.0 \%$, indicating the covariates were well balanced (Figure 3). As shown in Table 3, weighted Cox regression still showed significantly lower risks of overall mortality (HR 0.50, 95\% CI, 0.25$0.99, \mathrm{P}=0.047$ ) in the NYHA class I/II group. Detailed characteristics after PS matching and overlap PS weighting are shown in Table S1 and Table S2.

\section{Discussion}

Our study shows that early surgery can improve the outcomes of patients with severe TR undergoing tricuspid replacement. Higher incidences of overall mortality were consistent between the PS-matched cohort and overlap PS weighted analysis, indicating that waiting until patients develop symptoms has a price.

Although TVR accounts for a very small proportion of all cardiac valve surgeries, its mortality rate is high (9). Isolated TV surgeries have increased in recent years as awareness of the morbidity and mortality associated with severe TR has increased (10). However, limited data exists regarding long-term mortality outcomes after TV replacement (11). Furthermore, some studies have questioned the effect of surgical treatment of severe TR, triggering a heated debate $(5,12,13)$. Current guideline indications for surgical TV intervention include TV repair and replacement at the time of left-sided valve surgery in symptomatic and asymptomatic severe TR patients. In asymptomatic patients with severe primary TR (stage C) and progressive RV dilation or systolic dysfunction, isolated tricuspid valve surgery may be considered (3). Some experts also propose that early intervention, before severe right ventricle dysfunction and pulmonary hypertension occurs, seems to improve postoperative outcomes (14). Jeganathan and colleagues (15) showed that patients with NYHA class III/IV had significantly worse outcomes than those in NYHA classes I and II. Another study showed that inhospital mortality significantly correlated with NYHA class, with no mortality in patients in NYHA classes I and II, $19 \%$ mortality in NYHA class III patients, and $55.6 \%$ mortality in NYHA class IV patients (6). In addition to inhospital mortality, our study followed up with patients for 20 years. One hundred and sixty-three of the 256 patients we studied were in preoperative NYHA functional classes III and IV, suggesting that surgical timing was late in these patients. Similarly, studies of surgical intervention based on NYHA classification in degenerative mitral valve disease also suggest that surgical intervention should not wait 
Table 1 Baseline demographics, clinical and laboratory characteristics by New York Heart Association class

\begin{tabular}{|c|c|c|c|}
\hline Variables & NYHA class III/IV $(n=163)$ & NYHA class I/II (n=93) & $P$ value \\
\hline Gender, male & 109 (66.9) & $42(45.2)$ & 0.001 \\
\hline Hypertension & $11(6.7)$ & $9(9.7)$ & 0.550 \\
\hline Diabetes & $5(3.1)$ & $2(2.2)$ & 0.973 \\
\hline Lung disease & $2(1.2)$ & $1(1.1)$ & 1.000 \\
\hline Stroke & $5(3.1)$ & $0(0.0)$ & 0.216 \\
\hline Hepatitis C & $7(4.3)$ & $1(1.1)$ & 0.294 \\
\hline Functional & $56(34.4)$ & $15(16.1)$ & 0.003 \\
\hline MVR & $58(35.6)$ & $12(12.9)$ & $<0.001$ \\
\hline MVP & $3(1.8)$ & $1(1.1)$ & 1.000 \\
\hline AVR & $16(9.8)$ & $6(6.5)$ & 0.489 \\
\hline TVR & $8(4.9)$ & $9(9.7)$ & 0.225 \\
\hline TVP & $30(18.4)$ & $5(5.4)$ & 0.006 \\
\hline Second time or more & $19(11.7)$ & $5(5.4)$ & 0.151 \\
\hline NYHA & & & $<0.001$ \\
\hline I & $0(0.0)$ & $1(1.1)$ & \\
\hline $\operatorname{PLT}\left(\times 10^{9} / \mathrm{L}\right)$ & $144.00[109.50,194.00]$ & $170.50[129.25,220.00]$ & 0.018 \\
\hline BUN (mmol/L) & $6.50[5.20,8.30]$ & $5.80[4.40,6.90]$ & 0.001 \\
\hline AST (U/L) & $30.00[24.00,35.00]$ & $23.00[19.25,29.75]$ & $<0.001$ \\
\hline ALT (U/L) & $20.00[15.75,29.25]$ & $23.00[16.00,34.00]$ & 0.139 \\
\hline TBil $(\mu \mathrm{mol} / \mathrm{L})$ & $26.75[15.48,31.40]$ & $23.50[14.00,27.30]$ & 0.024 \\
\hline Hypoproteinemia & $25(17.6)$ & $11(14.7)$ & 0.718 \\
\hline $\mathrm{EF}(\%)$ & $62.0[55.5,67.0]$ & $63.0[60.0,68.0]$ & 0.101 \\
\hline Liver enlargements & $37(22.7)$ & $5(5.4)$ & 0.001 \\
\hline Spleen enlargement & $35(21.5)$ & $6(6.5)$ & 0.003 \\
\hline Ascites & $6(3.7)$ & $1(1.1)$ & 0.402 \\
\hline Lower extremity edema & $69(42.3)$ & $15(16.1)$ & $<0.001$ \\
\hline
\end{tabular}

Values are expressed as median with interquartile range or number (percentage). NYHA, New York Heart Association; BMI, body mass index; AF, atrial fibrillation; MVR, mitral valve replacement; MVP, mitral valvuloplasty; AVR, aortic valve replacement; TVR, tricuspid valve replacement; TVP, tricuspid valvuloplasty; WBC, white blood cell; PLT, platelet; BUN, blood urea nitrogen; AST, aspartate aminotransferase; ALT, alanine aminotransferase; TBil, total bilirubin; EF, ejection fraction. 
Table 2 Surgical information and clinical outcomes

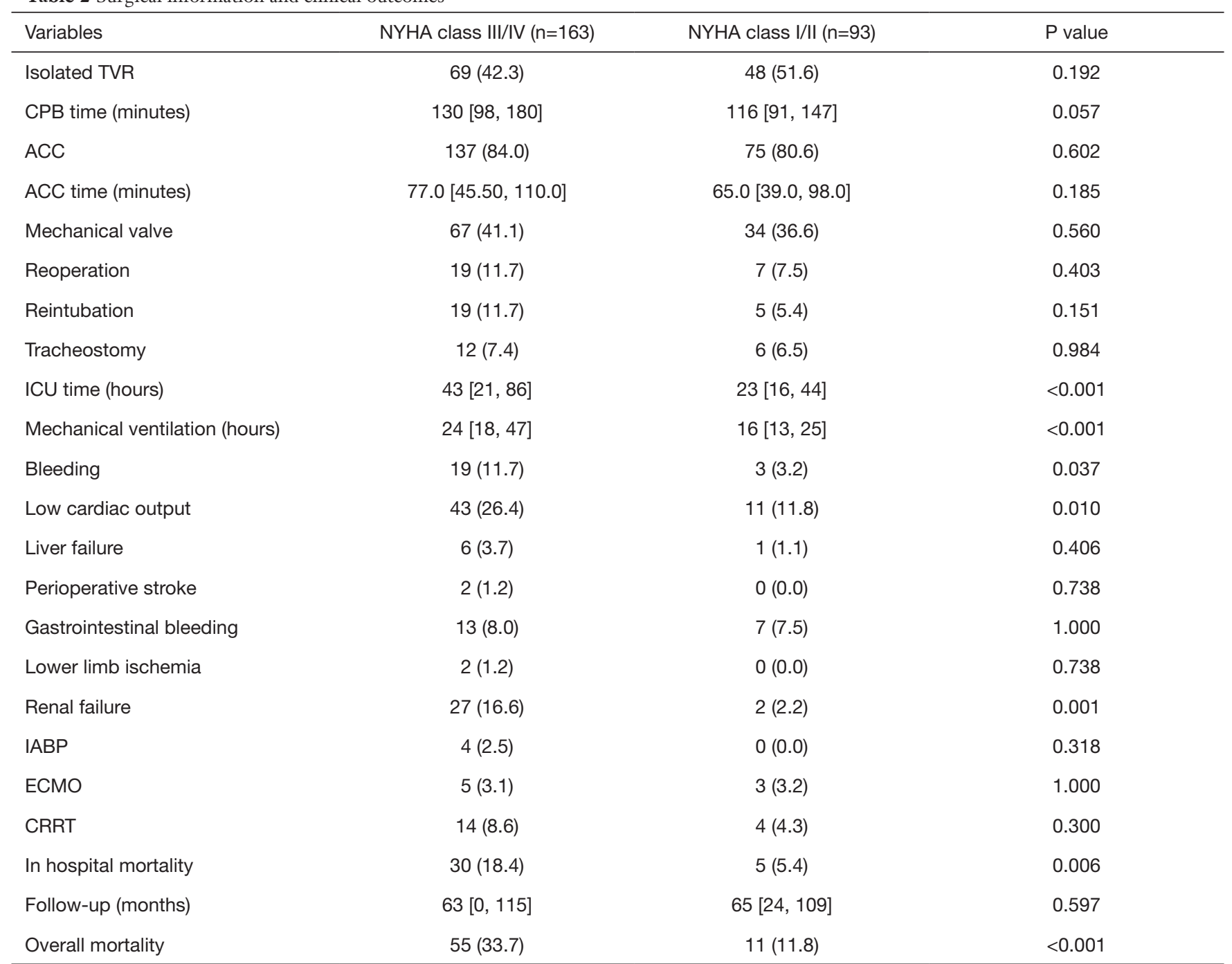

Values are expressed as median with interquartile range or number (percentage). NYHA, New York Heart Association; CPB, cardiopulmonary bypass; ACC, aortic cross clamp; ICU, intensive care unit; IABP, intra-aortic balloon pump; ECMO, extracorporeal Membrane Oxygenation; CRRT, continuous renal replacement therapy.

until symptoms develop (16). Anand and colleagues (7) published a detailed study of asymptomatic degenerative mitral regurgitation repair and found that overall survival significantly increased in patients designated NYHA I compared to those designated NYHA II and III/IV $(\mathrm{P}<0.001)$. Given this result and the fact that there was only one NYHA class I patient in our cohort, we divided the study population into an early surgery group (NYHA I/II) and a control group (NYHA III/IV).

The tricuspid valve is a complex anatomical structure whose disease, dysfunction and weighty role in right ventricular (RV) failure require better understanding, prompting increased interest in tricuspid valve surgery. The development of TR entails further RV dilatation and dysfunction, tricuspid annulus dilatation, tricuspid leaflet tethering and worsening of subsequent TR, perpetuating a vicious cycle whereby TR feeds more TR (17). The presence of TR in the setting of RV failure indicates a poor prognosis. In our study, patients in the NYHA class I/II group exhibited fewer signs of RHF, such as liver and spleen enlargements, ascites and lower extremity edema. In addition, patients in this group presented fewer comorbidities, such as histories of $\mathrm{AF}$ and previous valve surgery. Umehara and colleagues (18) considered the 


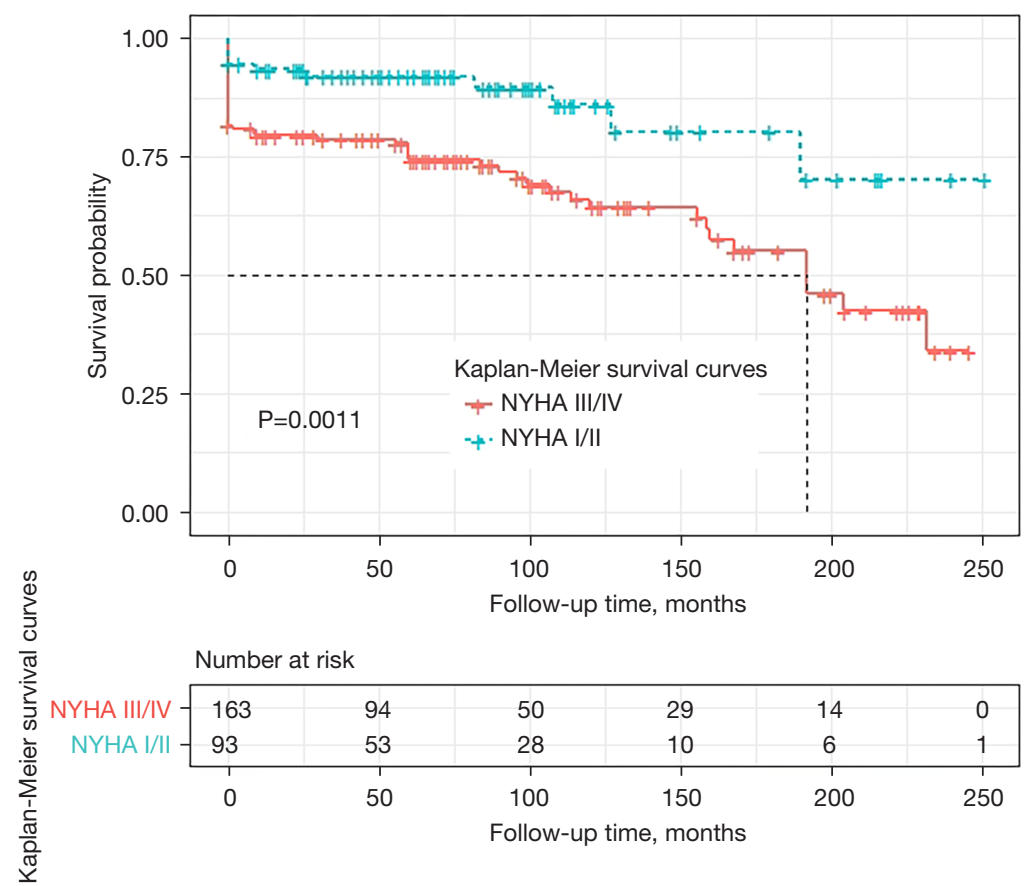

Figure 2 Kaplan-Meier curves with corresponding numbers at-risk for overall survival by NYHA class. NYHA, New York Heart Association.

Table 3 Summary of clinical outcomes and hazard ratio for NYHA classification

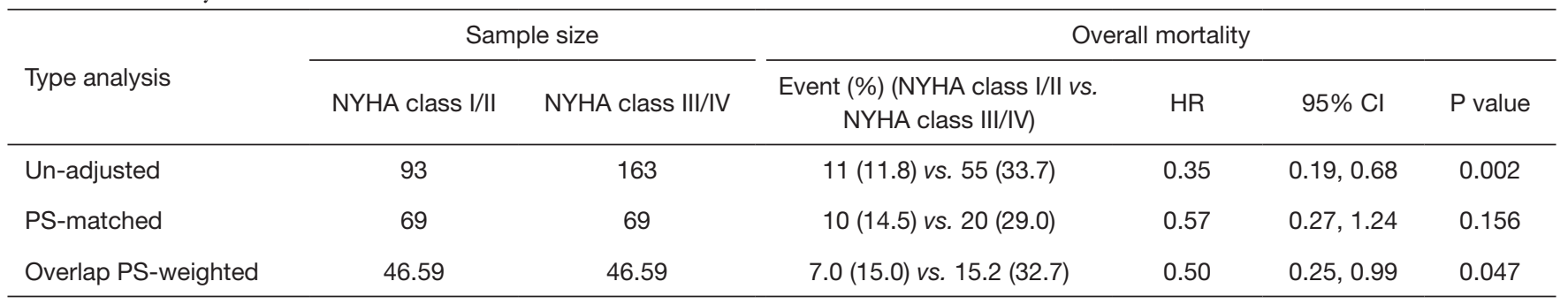

NYHA, New York Heart Association; PS, propensity score; HR, hazard ratio; Cl, confidence interval.

surgical outcomes following reoperative tricuspid surgery unsatisfactory. It is also well known that TV surgery is associated with poor long-term outcomes due to multiple patient comorbidities (19). Early surgical correction of TR appears to provide significantly lower operative risks.

The present study employed a very heterogeneous and complex group of patients for which choosing the best treatment may be challenging, especially as the majority of patients present late, when surgical intervention is often associated with significant periprocedural morbidity and mortality (20). Many doctors want to proceed before the onset of AF, symptoms, or RV dysfunction in these patients. However, the substantial surgical risks and the reality of high mortality have often prevented this decision. Interestingly, while some patients with organic mitral regurgitation report that they have no symptoms, follow-up exercise testing after repair has documented improved peak oxygen performance and maximal workload (21), indicating that early surgery may result in long-term patient benefits. Applying the overlap PS method for verification, our realworld study provides conclusions regarding long-term prognoses that should prompt surgeons to consider treating patients with asymptomatic or mildly symptomatic severe TR. On the other hand, a number of novel transcatheter tricuspid valve interventions have emerged over the past decade, which may benefit some severe TR patients (22). 


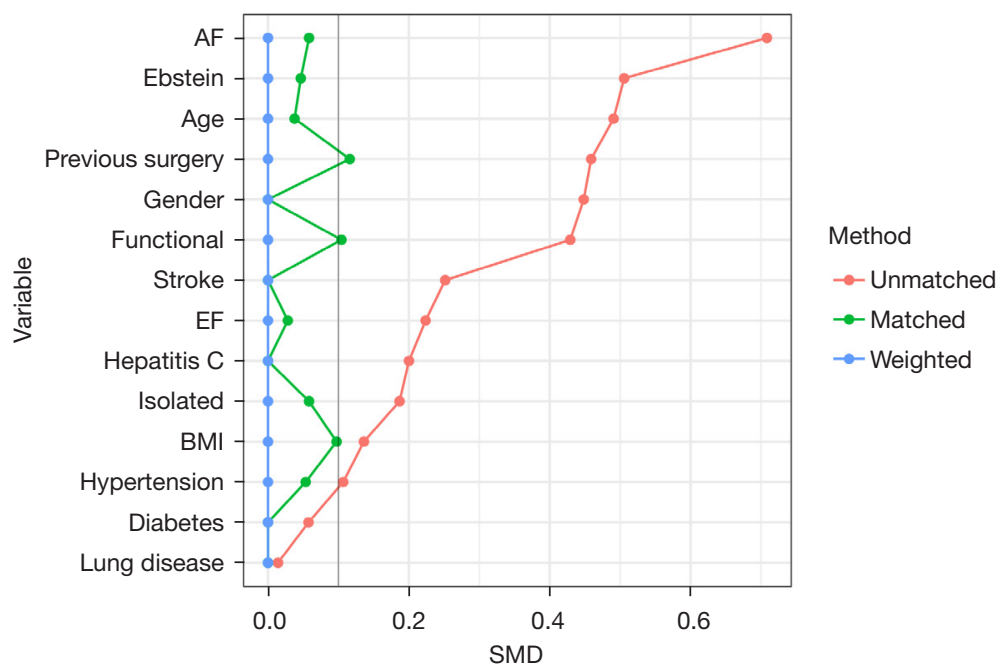

Figure 3 Standardized mean differences for NYHA pairwise comparisons. AF, atrial fibrillation; BMI, body mass index; EF, ejection fraction; NYHA, New York Heart Association; SMD, standardized mean difference.

There are several limitations to our study. First, the study has the usual limitations of retrospective investigations and may not be widely generalizable. The study also was not randomized, and the sample size was limited, though we applied overlap PS weights to make up for this as much as possible. In addition, we only included patients undergoing TVR, not patients undergoing tricuspid valve repair, which limits our conclusions. Furthermore, this is a study of nearly two decades, and missing clinical data, including ultrasound and magnetic resonance imaging, restricts assessment of right heart function. Finally, we only compared early surgery patients to patients in NYHA class III/IV, and we did not compare early surgery patients to the conservative treatment group.

\section{Conclusions}

Our study suggests that surgery for severe TR patients should be considered before the development of advanced HF, when patients are asymptomatic or mildly symptomatic, supporting the validity of a recent clinical trend toward earlier and more aggressive TR intervention.

\section{Acknowledgments}

Funding: This study was supported by Beijing Hospitals Authority Youth Program (QML20170605) \& Scientific Research Common Program of Beijing Municipal Commission of education (KM201910025013).

\section{Footnote}

Reporting Checklist: The authors have completed the STROBE reporting checklist. Available at https://dx.doi. org/10.21037/cdt-21-311

Data Sharing Statement: Available at https://dx.doi. org/10.21037/cdt-21-311

Conflicts of Interest: All authors have completed the ICMJE uniform disclosure form (available at https://dx.doi. org/10.21037/cdt-21-311). The authors have no conflicts of interest to declare.

Ethical Statement: The authors are accountable for all aspects of the work in ensuring that questions related to the accuracy or integrity of any part of the work are appropriately investigated and resolved. The study was conducted in accordance with the Declaration of Helsinki (as revised in 2013). The study was approved by Institutional Review Board of Beijing Anzhen Hospital (NO.: 2020101X) and individual consent for this retrospective analysis was waived.

Open Access Statement: This is an Open Access article distributed in accordance with the Creative Commons Attribution-NonCommercial-NoDerivs 4.0 International License (CC BY-NC-ND 4.0), which permits the noncommercial replication and distribution of the article with 
the strict proviso that no changes or edits are made and the original work is properly cited (including links to both the formal publication through the relevant DOI and the license). See: https://creativecommons.org/licenses/by-nc-nd/4.0/.

\section{References}

1. Bohbot Y, Chadha G, Delabre J, et al. Characteristics and prognosis of patients with significant tricuspid regurgitation. Arch Cardiovasc Dis 2019;112:604-14.

2. Nath J, Foster E, Heidenreich PA. Impact of tricuspid regurgitation on long-term survival. J Am Coll Cardiol 2004;43:405-9.

3. Writing Committee Members, Otto CM, Nishimura RA, et al. 2020 ACC/AHA Guideline for the Management of Patients With Valvular Heart Disease: A Report of the American College of Cardiology/American Heart Association Joint Committee on Clinical Practice Guidelines. 2021 Mar 9;77(9):1275]. J Am Coll Cardiol 2021;77:e25-e197. Erratum in: J Am Coll Cardiol. 2021 Feb 2;77(4):509. doi: 10.1016/j.jacc.2020.12.040. Epub 2020 Dec 28. J Am Coll Cardiol. 2021 Mar 9;77(9):1275. doi: 10.1016/j.jacc.2021.02.007.

4. Baumgartner H, Falk V, Bax JJ, et al. 2017 ESC/EACTS Guidelines for the management of valvular heart disease. Eur Heart J 2017;38:2739-91.

5. Axtell AL, Bhambhani V, Moonsamy P, et al. Surgery Does Not Improve Survival in Patients With Isolated Severe Tricuspid Regurgitation. J Am Coll Cardiol 2019;74:715-25.

6. Litwiński P, Kołsut P, Sitko T, et al. Results and factors associated with adverse outcome after tricuspid valve replacement. Kardiol Pol 2018;76:731-9.

7. Desai A, Thomas JD, Bonow RO, et al. Asymptomatic degenerative mitral regurgitation repair: Validating guidelines for early intervention. J Thorac Cardiovasc Surg 2021;161:981-994.e5.

8. Thomas LE, Li F, Pencina MJ. Overlap Weighting: A Propensity Score Method That Mimics Attributes of a Randomized Clinical Trial. JAMA 2020;323:2417-8.

9. Wong WK, Chen SW, Chou AH, et al. Late Outcomes of Valve Repair Versus Replacement in Isolated and Concomitant Tricuspid Valve Surgery: A Nationwide Cohort Study. J Am Heart Assoc 2020;9:e015637.

10. Vassileva CM, Shabosky J, Boley T, et al. Tricuspid valve surgery: the past 10 years from the Nationwide Inpatient Sample (NIS) database. J Thorac Cardiovasc Surg 2012;143:1043-9.
11. Ingraham BS, Pislaru SV, Nkomo VT, et al. Characteristics and treatment strategies for severe tricuspid regurgitation. Heart 2019;105:1244-50.

12. O'Gara PT, Gillinov M, Rodés-Cabau J. Surgery for Isolated Tricuspid Regurgitation: Limitations of Observational Analyses. J Am Coll Cardiol 2019;74:726-8.

13. Miura M, Taramasso $M$, Gavazzoni $M$, et al. What Is the Best Option in Patients With Isolated Severe Tricuspid Regurgitation? J Am Coll Cardiol 2019;74:2829.

14. Tagliari AP, Perez-Camargo D, Taramasso M. Tricuspid regurgitation: when is it time for surgery? Expert Rev Cardiovasc Ther 2021;19:47-59.

15. Jeganathan R, Armstrong S, Al-Alao B, et al. The risk and outcomes of reoperative tricuspid valve surgery. Ann Thorac Surg 2013;95:119-24.

16. Gillinov AM, Mihaljevic T, Blackstone EH, et al. Should patients with severe degenerative mitral regurgitation delay surgery until symptoms develop? Ann Thorac Surg 2010;90:481-8.

17. Asmarats L, Taramasso M, Rodés-Cabau J. Tricuspid valve disease: diagnosis, prognosis and management of a rapidly evolving field. Nat Rev Cardiol 2019;16:538-54.

18. Umehara N, Miyata H, Motomura N, et al. Surgical results of reoperative tricuspid surgery: analysis from the Japan Cardiovascular Surgery Database†. Interact Cardiovasc Thorac Surg 2014;19:82-7.

19. Saran N, Dearani JA, Said SM, et al. Long-term outcomes of patients undergoing tricuspid valve surgery†. Eur J Cardiothorac Surg 2019;56:950-8.

20. Latib A, Grigioni F, Hahn RT. Tricuspid regurgitation: what is the real clinical impact and how often should it be treated? EuroIntervention 2018;14:AB101-AB111.

21. Madaric J, Watripont P, Bartunek J, et al. Effect of mitral valve repair on exercise tolerance in asymptomatic patients with organic mitral regurgitation. Am Heart J 2007;154:180-5.

22. Asmarats L, Puri R, Latib A, et al. Transcatheter Tricuspid Valve Interventions: Landscape, Challenges, and Future Directions. J Am Coll Cardiol 2018;71:2935-56.

Cite this article as: Hua K, Zhao R, Peng Z, Yang Y, Florian O, Mao B, Yang X. Early surgery can improve the outcomes of patients with severe tricuspid regurgitation undergoing tricuspid replacement. Cardiovasc Diagn Ther 2021;11(5):1058-1066. doi: 10.21037/cdt-21-311 
Table S1 PS-matched groups: characteristics and outcomes

\begin{tabular}{|c|c|c|c|c|}
\hline Variables & NYHA class III/IV ( $n=69)$ & NYHA class I/II $(n=69)$ & $P$ value & SMD \\
\hline Age (years) & $43.00[33.00,55.00]$ & $42.00[30.00,54.00]$ & 0.777 & 0.038 \\
\hline Gender, male & $31(44.9)$ & $31(44.9)$ & 1.000 & 0 \\
\hline $\mathrm{BMI}\left(\mathrm{kg} / \mathrm{m}^{2}\right)$ & $22.13[19.47,23.89]$ & $22.49[20.57,24.68]$ & 0.204 & 0.097 \\
\hline Hypertension & $6(8.7)$ & $5(7.2)$ & 1.000 & 0.054 \\
\hline Diabetes & $1(1.4)$ & $1(1.4)$ & 1.000 & 0 \\
\hline $\mathrm{AF}$ & $37(53.6)$ & $39(56.5)$ & 0.864 & 0.058 \\
\hline Lung disease & $1(1.4)$ & $1(1.4)$ & 1.000 & 0 \\
\hline Stroke & $0(0)$ & $0(0)$ & 1.000 & 0 \\
\hline Hepatitis C & $1(1.4)$ & $1(1.4)$ & 1.000 & 0 \\
\hline Functional & $17(24.6)$ & $14(20.3)$ & 0.683 & 0.104 \\
\hline Rheumatic & $37(53.6)$ & $27(39.1)$ & 0.124 & 0.294 \\
\hline Ebstein's anomaly & $7(10.1)$ & $8(11.6)$ & 1.000 & 0.047 \\
\hline Previous valve surgery & $36(52.2)$ & $32(46.4)$ & 0.609 & 0.116 \\
\hline MVR & $19(27.5)$ & $12(17.4)$ & 0.221 & 0.245 \\
\hline MVP & $1(1.4)$ & $1(1.4)$ & 1 & 0 \\
\hline AVR & $2(2.9)$ & $6(8.7)$ & 0.274 & 0.25 \\
\hline TVR & $3(4.3)$ & $9(13.0)$ & 0.131 & 0.312 \\
\hline TVP & $12(17.4)$ & $4(5.8)$ & 0.063 & 0.368 \\
\hline Second time or more & $8(11.6)$ & $5(7.2)$ & 0.56 & 0.149 \\
\hline NYHA & & & $<0.001$ & 11.696 \\
\hline I & $0(0.0)$ & $1(1.4)$ & & \\
\hline$\|$ & $0(0.0)$ & $68(98.6)$ & & \\
\hline III & $53(76.8)$ & $0(0.0)$ & & \\
\hline IV & $16(23.2)$ & $0(0.0)$ & & \\
\hline Hemoglobin & $131.0[118.5,145.5]$ & $137.0[121.5,148.5]$ & 0.317 & 0.187 \\
\hline WBC $\left(\times 10^{9} / L\right)$ & $5.38[4.40,6.60]$ & $5.60[4.27,6.85]$ & 0.669 & 0.135 \\
\hline $\operatorname{PLT}\left(\times 10^{9} / \mathrm{L}\right)$ & $150.0[117.0,203.5]$ & $159.0[120.5,207.5]$ & 0.599 & 0.065 \\
\hline BUN (mmol/L) & $6.50[5.25,7.83]$ & $5.75[4.50,6.68]$ & 0.009 & 0.473 \\
\hline AST (U/L) & $28.00[24.00,35.00]$ & $23.50[20.75,30.25]$ & 0.003 & 0.426 \\
\hline ALT (U/L) & $22.0[16.0,31.0]$ & $22.0[16.0,38.0]$ & 0.973 & 0.126 \\
\hline TBil $(\mu \mathrm{mol} / \mathrm{L})$ & $27.30[18.80,34.58]$ & $23.70[15.45,27.30]$ & 0.03 & 0.401 \\
\hline Hypoproteinemia & $6(10.2)$ & $10(17.5)$ & 0.378 & 0.215 \\
\hline $\mathrm{EF}(\%)$ & $63.0[57.0,68.0]$ & $63.0[57.0,68.0]$ & 0.821 & 0.028 \\
\hline Liver enlargements & $9(13.0)$ & $5(7.2)$ & 0.398 & 0.193 \\
\hline Spleen enlargement & $8(11.6)$ & $6(8.7)$ & 0.778 & 0.096 \\
\hline Ascites & $0(0.0)$ & $1(1.4)$ & 1.000 & 0.171 \\
\hline Lower extremity edema & $27(39.1)$ & $14(20.3)$ & 0.025 & 0.421 \\
\hline Isolated TVR & $30(43.5)$ & $32(46.4)$ & 0.864 & 0.058 \\
\hline CPB time (minutes) & $130.0[101.0,176.0]$ & $116.0[91.0,155.0]$ & 0.077 & 0.313 \\
\hline ACC & $62(89.9)$ & $54(78.3)$ & 0.104 & 0.321 \\
\hline ACC time (minutes) & $81.0[53.0,111.0]$ & $67.0[36.0,99.0]$ & 0.166 & 0.256 \\
\hline Mechanical valve & $31(44.9)$ & $27(39.1)$ & 0.605 & 0.118 \\
\hline Reoperation & $7(10.1)$ & $5(7.2)$ & 0.763 & 0.103 \\
\hline Reintubation & $7(10.1)$ & $4(5.8)$ & 0.530 & 0.161 \\
\hline Tracheostomy & $4(5.8)$ & $5(7.2)$ & 1.000 & 0.059 \\
\hline ICU time (hours) & $38.0[21.0,73.0]$ & $24.0[18.0,60.0]$ & 0.062 & 0.098 \\
\hline Mechanical ventilation (hours) & $20.0[18.0,42.0]$ & $18.0[14.0,31.0]$ & 0.020 & 0.08 \\
\hline Bleeding & $9(13.0)$ & $2(2.9)$ & 0.059 & 0.381 \\
\hline Low cardiac output & $13(18.8)$ & $9(13.0)$ & 0.485 & 0.159 \\
\hline Liver failure & $2(2.9)$ & $1(1.4)$ & 1.000 & 0.100 \\
\hline Perioperative stroke & $1(1.4)$ & $0(0.0)$ & 1.000 & 0.171 \\
\hline Gastrointestinal bleeding & $3(4.3)$ & $6(8.7)$ & 0.490 & 0.177 \\
\hline Lower limb ischemia & $0(0)$ & $0(0)$ & 1.000 & 0 \\
\hline Renal failure & $10(14.5)$ & $2(2.9)$ & 0.034 & 0.420 \\
\hline IABP & $0(0)$ & $0(0)$ & 1.000 & 0 \\
\hline ECMO & $0(0.0)$ & $3(4.3)$ & 0.243 & 0.302 \\
\hline CRRT & $3(4.3)$ & $4(5.8)$ & 1.000 & 0.066 \\
\hline In hospital mortality (\%) & $10(14.5)$ & $5(7.2)$ & 0.274 & 0.234 \\
\hline Follow up (months) & $66.0[9.0,134.0]$ & $65.0[14.0,104.0]$ & 0.397 & 0.216 \\
\hline Overall mortality (\%) & $20(29.0)$ & $10(14.5)$ & 0.063 & 0.357 \\
\hline
\end{tabular}

Values are expressed as median with interquartile range or number (percentage). NYHA, New York Heart Association; SMD, standardized mean difference; BMI, body mass index; AF, atrial fibrillation; MVR, mitral valve replacement; MVP, mitral valvuloplasty; AVR, aortic valve replacement; TVR, tricuspid valve replacement; TVP, tricuspid valvuloplasty; WBC, white blood cell; PLT, platelet; TBil, total bilirubin; $\mathrm{EF}$, ejection fraction; CPB, cardiopulmonary bypass; ACC, aortic cross clamp; ICU, intensive care unit; IABP, intra-aortic balloon pump; ECMO, extracorporeal membrane oxygenation; CRRT, continuous renal replacement therapy. 
Table S2 Overlap PS-weighted groups: characteristics and outcomes

\begin{tabular}{|c|c|c|c|c|}
\hline Variables & NYHA class III/IV (n=69) & NYHA class I/II (n=69) & $P$ value & SMD \\
\hline Age (years) & $43.00[33.00,55.00]$ & $42.00[31.16,53.78]$ & 0.910 & $<0.001$ \\
\hline Gender, male & $24.3(52.2)$ & $24.3(52.2)$ & 1.000 & 0 \\
\hline $\mathrm{BMI}\left(\mathrm{kg} / \mathrm{m}^{2}\right)$ & $21.88[19.63,24.13]$ & $22.22[20.29,24.36]$ & 0.438 & $<0.001$ \\
\hline Hypertension & $3.9(8.3)$ & $3.9(8.3)$ & 1.000 & 0 \\
\hline Diabetes & $1.1(2.4)$ & $1.1(2.4)$ & 1.000 & 0 \\
\hline AF & $27.4(58.8)$ & $27.4(58.8)$ & 1.000 & 0 \\
\hline Lung disease & $0.6(1.3)$ & $0.6(1.3)$ & 1.000 & 0 \\
\hline Stroke & $0.0(0.0)$ & $0.0(0.0)$ & 0.046 & 0 \\
\hline Hepatitis C & $0.8(1.6)$ & $0.8(1.6)$ & 1.000 & 0 \\
\hline Functional & $10.7(22.9)$ & $10.7(22.9)$ & 1.000 & 0 \\
\hline Rheumatic & $24.2(52.0)$ & $19.4(41.7)$ & 0.169 & 0.208 \\
\hline Ebstein's anomaly & $4.7(10.1)$ & $4.7(10.1)$ & 1.000 & 0 \\
\hline Previous valve surgery & $22.2(47.7)$ & $22.2(47.7)$ & 1.000 & 0 \\
\hline MVR & $10.9(23.5)$ & $8.9(19.1)$ & 0.470 & 0.108 \\
\hline MVP & $0.2(0.4)$ & $0.5(1.0)$ & 0.359 & 0.082 \\
\hline AVR & $2.4(5.2)$ & $4.6(9.8)$ & 0.179 & 0.176 \\
\hline TVR & $2.8(6.0)$ & $5.4(11.5)$ & 0.186 & 0.195 \\
\hline TVP & $7.0(14.9)$ & $2.4(5.1)$ & 0.031 & 0.331 \\
\hline Second time or more & $4.3(9.1)$ & $3.4(7.3)$ & 0.665 & 0.066 \\
\hline NYHA & & & $<0.001$ & 12.563 \\
\hline I & $0.0(0.0)$ & $0.6(1.3)$ & & \\
\hline$\|$ & $0.0(0.0)$ & $46.0(98.7)$ & & \\
\hline III & $37.7(80.9)$ & $0.0(0.0)$ & & \\
\hline IV & $8.9(19.1)$ & $0.0(0.0)$ & & \\
\hline Hemoglobin & $130.00[116.00,146.00]$ & $133.95[118.68,144.00]$ & 0.605 & 0.090 \\
\hline WBC $\left(\times 10^{9} / \mathrm{L}\right)$ & $5.34[4.47,6.60]$ & $5.39[4.22,6.65]$ & 0.817 & 0.188 \\
\hline PLT (×109/L) & $156.78[113.00,209.00]$ & $159.00[113.76,214.67]$ & 0.982 & 0.054 \\
\hline BUN (mmol/L) & $6.40[5.20,8.20]$ & $6.03[4.48,6.80]$ & 0.005 & 0.378 \\
\hline AST (U/L) & $29.0[24.0,35.0]$ & $24.0[20.0,30.0]$ & $<0.001$ & 0.43 \\
\hline ALT (U/L) & $21.17[16.00,30.00]$ & $22.00[16.00,33.63]$ & 0.695 & 0.101 \\
\hline TBil $(\mu \mathrm{mol} / \mathrm{L})$ & $27.30[18.17,33.20]$ & $23.61[14.11,27.30]$ & 0.008 & 0.438 \\
\hline Hypoproteinemia & $5.3(13.4)$ & $6.4(17.2)$ & 0.499 & 0.107 \\
\hline EF (\%) & $63.0[57.0,68.0]$ & $63.0[57.0,67.0]$ & 0.855 & $<0.001$ \\
\hline Liver enlargements & $9.1(19.6)$ & $3.3(7.1)$ & 0.021 & 0.373 \\
\hline Spleen enlargement & $7.9(16.9)$ & $4.1(8.7)$ & 0.114 & 0.245 \\
\hline Ascites & $1.0(2.1)$ & $0.9(1.9)$ & 0.909 & 0.017 \\
\hline Lower extremity edema & $16.8(36.1)$ & $9.7(20.7)$ & 0.026 & 0.345 \\
\hline Isolated TVR & $20.6(44.2)$ & $20.6(44.2)$ & 1.000 & 0 \\
\hline CPB time (minutes) & $125.00[97.55,172.00]$ & $115.44[91.51,147.00]$ & 0.114 & 0.262 \\
\hline ACC & $40.6(87.1)$ & $37.2(79.8)$ & 0.152 & 0.196 \\
\hline ACC time (minutes) & $75.72[47.72,103.31]$ & $65.77[38.18,96.58]$ & 0.175 & 0.221 \\
\hline Mechanical valve & $21.6(46.4)$ & $16.7(35.9)$ & 0.153 & 0.213 \\
\hline Reoperation & $4.7(10.0)$ & $3.3(7.1)$ & 0.484 & 0.105 \\
\hline Reintubation & $5.2(11.2)$ & $2.4(5.2)$ & 0.148 & 0.221 \\
\hline Tracheostomy & $2.4(5.1)$ & $4.0(8.5)$ & 0.317 & 0.137 \\
\hline ICU time (hours) & $41.71[20.00,77.43]$ & $27.20[17.00,61.83]$ & 0.022 & 0.074 \\
\hline Mechanical ventilation (hours) & $21.00[17.00,44.84]$ & $18.00[14.00,33.03]$ & 0.002 & 0.018 \\
\hline Bleeding & $4.3(9.2)$ & $1.7(3.7)$ & 0.144 & 0.227 \\
\hline Low cardiac output & $10.9(23.3)$ & $6.9(14.7)$ & 0.159 & 0.220 \\
\hline Liver failure & $1.7(3.7)$ & $0.8(1.8)$ & 0.532 & 0.113 \\
\hline Perioperative stroke & $0.3(0.7)$ & $0.0(0.0)$ & 0.165 & 0.120 \\
\hline Gastrointestinal bleeding & $3.2(6.9)$ & $4.1(8.8)$ & 0.622 & 0.072 \\
\hline Lower limb ischemia & $0.6(1.2)$ & $0.0(0.0)$ & 0.233 & 0.158 \\
\hline Renal failure & $7.2(15.5)$ & $1.2(2.6)$ & 0.006 & 0.460 \\
\hline IABP & $0.6(1.3)$ & $0.0(0.0)$ & 0.100 & 0.165 \\
\hline ECMO & $0.7(1.4)$ & $2.3(5.0)$ & 0.096 & 0.201 \\
\hline CRRT & $3.1(6.6)$ & $2.8(6.1)$ & 0.887 & 0.022 \\
\hline In hospital mortality (\%) & $8.0(17.2)$ & $3.4(7.4)$ & 0.072 & 0.302 \\
\hline Follow-up (months) & $65.00[7.75,128.78]$ & $66.06[22.22,108.17]$ & 0.729 & 0.137 \\
\hline Overall mortality (\%) & $15.2(32.7)$ & $7.0(15.0)$ & 0.009 & 0.424 \\
\hline
\end{tabular}

Values are expressed as median with interquartile range or number (percentage). NYHA, New York Heart Association; SMD, standardized mean difference; BMI, body mass index; AF, atrial fibrillation; MVR, mitral valve replacement; MVP, mitral valvuloplasty; AVR, aortic valve replacement; TVR, tricuspid valve replacement; TVP, tricuspid valvuloplasty; WBC, white blood cell; PLT, platelet; TBil, total bilirubin; $E F$, ejection fraction. CPB, cardiopulmonary bypass; ACC, aortic cross clamp; ICU, intensive care unit; IABP, intra-aortic balloon pump; ECMO, extracorporeal membrane oxygenation; CRRT, continuous renal replacement therapy. 